\title{
Percolation effects in the capacitive properties of metal-oxide varistors in the range of high voltage
}

\author{
A. S. Tonkoshkur*, I. V. Gomilko and A. Yu Lyashkov \\ Department of Physics, Electronics and Computer Systems, Dniepropetrovsk \\ National University, Gagarin av. 72, Dniepropetrovsk, 49010, Ukraine \\ *vdnu@yandex.ru
}

Received 16 February 2014; Revised 1 May 2014; Accepted 12 May 2014; Published 10 June 2014

\begin{abstract}
$\mathrm{C}-\mathrm{V}$ characteristics of $\mathrm{ZnO}$-based ceramic structures used in manufacturing high-voltage and low-voltage varistors of different chemical compositions and manufacturing techniques have been investigated. A correlation between the intensity of electric field corresponding to transition of the $C-V$ characteristics to the negative capacitances and average sizes of grains of a varistor structure has been established. Obtained data have been interpreted with the use of notions of the percolation theory of electric conductivity. The Shklovskii-De Gennes model has been used. It has been shown that on the highly nonlinear segment of $C-V$ characteristics of a varistor structure, the size of an infinite cluster are limited to several intercrystallite potential barriers. This result is observed in all kinds of investigated varistor ceramics.
\end{abstract}

Keywords: Varistor; ceramics; $C-V$ characteristics; capacitance; percolation.

\section{Introduction}

$\mathrm{ZnO}$ varistor ceramics is one of the typical examples of nonhomogeneous structures with intercrystallite potential barriers (IPB). One of its specific properties is field dependence of dielectric permittivity $\varepsilon$, which, as the applied electrostatic field increases, passes three distinctive areas: decrease (or steadiness) of $\varepsilon$ (area 1), its growth (2) and abrupt decline with passing to negative values (3). ${ }^{1-3}$

Field dependence of the high frequency $\varepsilon$ (or capacitance $C$ ) in area 1 is interpreted in the framework of deformation of the Schottky double-barrier by the electric field. ${ }^{3,4}$ This effect is one of the main methods for determining parameters of metal-oxide varistors (in particular, the height of the intercrystallite potential barrier and concentration of donors) in studying the influence of admixtures (additives) or appearance of defects in the structure of the ceramics caused by some or other peculiarities of manufacturing technologies or by the process of degrading. ${ }^{5-9}$

Interpretation of the effect of negative capacitance is being discussed until now. In particular, there are known ideas of fast growth of conductivity in the area of breakdown, which stipulates bridging and fast fall of capacitance, ${ }^{1}$ of appearance of an inversion layer in the reversely shifted area of the spatial charge of the intercrystallite barrier in case of strong bends of energetic zones, ${ }^{10,11}$ of tunnel generation, stimulated by absorption of phonons, ${ }^{3}$ of generation of "hot" electrons overcoming the intercrystallite boundary that are capable of impact ionization and creation of holes in its depleted part, further recombination of which leads to appearance of inductance properties. ${ }^{2,12-14}$

Existing methods of analysis of $C-V$ characteristics of metal-oxide varistors are based on the model of a single intercrystallite potential barrier ("microvaristor"). With such immediate expansion of these dependences over the whole varistor structure, its electrical irregularity is not taken into account. It is worth mentioning that at present, besides the aforementioned "microvaristor" model other ways of taking into account the irregularity of varistor ceramics and its influence on electrical characteristics are known. These are the effective medium method, ${ }^{15}$ the use of the grid model based on Kirchhof"s equations, ${ }^{16}$ and also the use of the percolation theory. ${ }^{17}$ The indicated approaches are mainly used for analysis of volt-ampere characteristics and practically are not used for the $C-V$ ones. The exception is, Ref. 18 where a formula obtained in the framework of the effective medium theory for low-voltage (area 1) of $C-V$ characteristics and an attempt of its analysis from the point of view of the percolation theory are used. ${ }^{19}$ As one of the causes of poor understanding of the processes of the phenomenon of negative capacitance, insufficient experimental research of relationship between it and irregularity of the varistor structure should be named.

In this paper, results of research in regularities of manifestation of structural parameters, in particular, sizes of $\mathrm{ZnO}$ grains in the bulk properties (in the area of negative capacitance) is obtained by comparison of samples of metal-oxide varistor ceramics of different chemical compositions and manufacturing techniques.

This is an Open Access article published by World Scientific Publishing Company. It is distributed under the terms of the Creative Commons Attribution 3.0 (CC-BY) License. Further distribution of this work is permitted, provided the original work is properly cited. 


\section{Samples and Research Technique}

In the work, zinc oxide ceramics of three types were used: commercial high-voltage ceramics for varistors $\mathrm{SN} 2-2 \mathrm{com}-$ posed of $\mathrm{ZnO}-\mathrm{Bi}_{2} \mathrm{O}_{3}-\mathrm{CoO}-\mathrm{Sb}_{2} \mathrm{O}_{3}-\mathrm{SnO}_{2}-\mathrm{MnO}-\mathrm{B}_{2} \mathrm{O}_{3}$ - (type 1), low-voltage ceramics used in multilayered varistors KEKO MVL (type 2) and experimental ceramics $\mathrm{ZnO}-$ $\mathrm{Bi}_{2} \mathrm{O}_{3}-\mathrm{TiO}_{2}-\mathrm{CoO}-\mathrm{MnO}_{2}-\mathrm{NiO}$ (type 3).

These ceramics have typical microstructure described in many research works. ${ }^{20,23,24}$ The sizes of grains were measured with the use of optic and electron microscopy.

Samples of the bulk type (1 and 3) were in the shape of flat round tablets. Samples of multilayered varistors (type 2) were of rectangular shape. Their microstructure of interlaced layers of $\mathrm{ZnO}$ ceramics (20 to $100 \mu \mathrm{m}$ ) and electrode layers are made of $\mathrm{AgPd}{ }^{22,23}$ Dimensions of the samples and their threshold voltage $V_{n}$ (voltage across the varistor measured at a given reference current $I_{n}=1 \mathrm{ma}$ DC) are presented in Table 1.

For investigation of $C-V$ characteristics, the measurement scheme described in Ref. 2 and 25 was used, which allows one to measure capacitance in wide range of direct shifting voltage $(0-700 \mathrm{~V})$ and frequencies of alternating current $(20 \mathrm{~Hz}-50 \mathrm{kHz})$.

We connected the sample to a source of direct current via an inductance. Its value was chosen to be sufficiently large in order to avoid influence of direct current circuit on the results of measurements under the alternating current. An alternating current bridge circuit was connected to the samples via a blocking capacitor, which did not let the direct current component to the bridge.

Monitoring of the direct voltage on the sample and the current through it was done with a voltmeter and a milliammeter, respectively.

For measuring the negative capacitance, an additional capacitor with capacitance of 1 to $10 \mu \mathrm{F}$ was connected in parallel to the sample. The total of this additional capacitance and the negative capacitance $C_{\sum}$ of the varistor was positive and provided the loss of conductivity sufficient for reliable measurements of the capacitance $\left(\operatorname{tg} \delta=G_{0} /\left(2 \pi \cdot f \cdot C_{\sum}\right)\right.$ $<1$, where $G_{0}$ is the conductance of the sample, $f$ is the frequency of the alternating electric field in the range of determination of the negative capacitance of the varistor).

Table 1. Parameters of samples of varistors.

\begin{tabular}{cccr}
\hline $\begin{array}{c}\text { Type of } \\
\text { ceramics }\end{array}$ & $\begin{array}{c}\text { Diameter or } \\
\text { dimensions, cm }\end{array}$ & Thickness, cm & $V_{n}, \mathrm{~V}$ \\
\hline 1 & 2.6 & $\sim 0.13$ & 137 \\
2 & $0.5 \times 0.57$ & $\begin{array}{c}0.1 \text { (total of } \\
\text { all layers) }\end{array}$ & 31 \\
3 & 8 & 0.1 & 50 \\
\hline
\end{tabular}

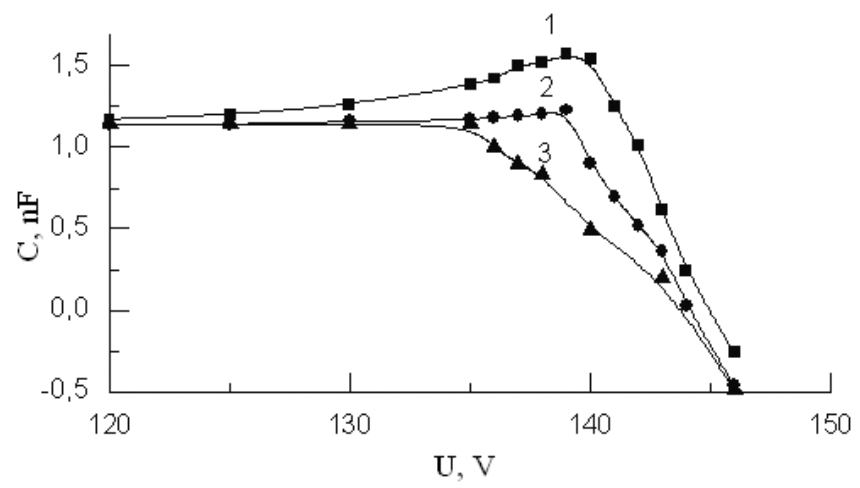

Fig. 1. $C-V$ characteristics of a sample of ceramics of type 1 at different frequencies, Hz: $1-10^{3}, 2-5 \times 10^{3}, 3-10^{4}$.

\section{Experimental $C-V$-characteristics of Ceramics}

The dependences of the capacitance $C$ of the ceramic samples under consideration of the applied direct voltage $V$ showed segments of weak change of the capacitance, its growth and abrupt decline to negative values with the growth of $V$, (Figs. 1 and 2), which corresponds with known published data. ${ }^{1-3,23}$ With the growth of frequency, at which the

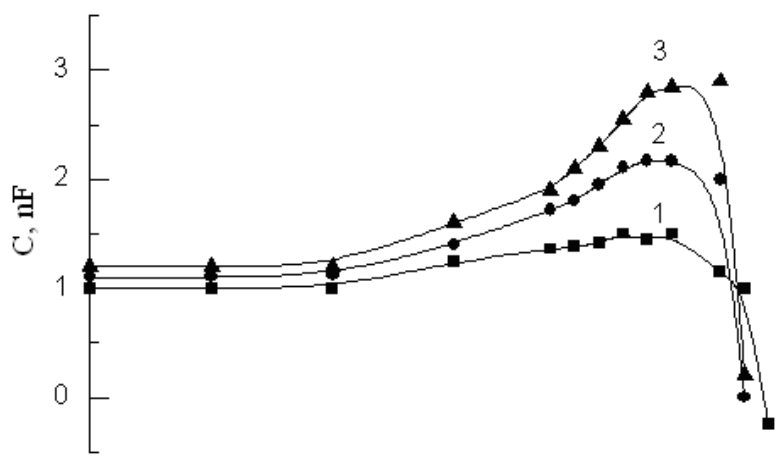

(a)

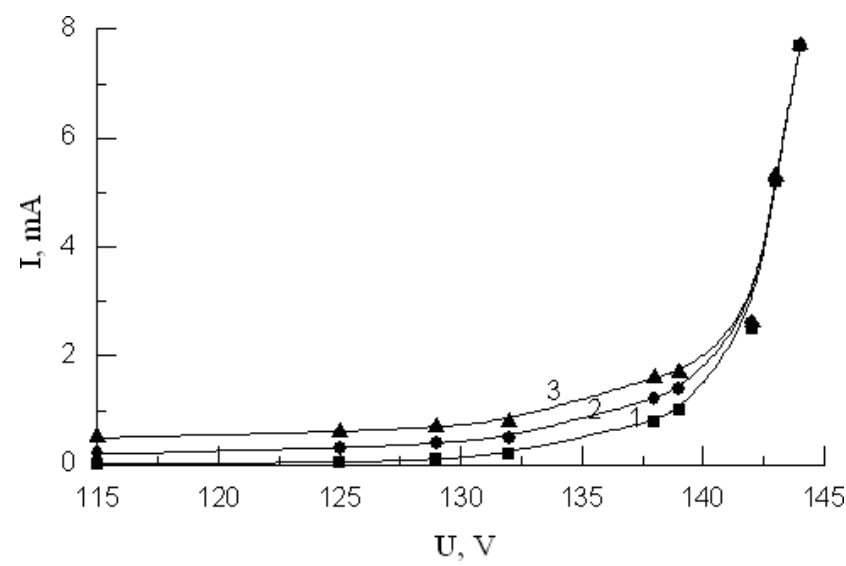

(b)

Fig. 2. Field dependence of capacitance (a) and current (b) of type 2 varistor ceramics at different temperatures, K: 1-300, 2-340, 3-360. 


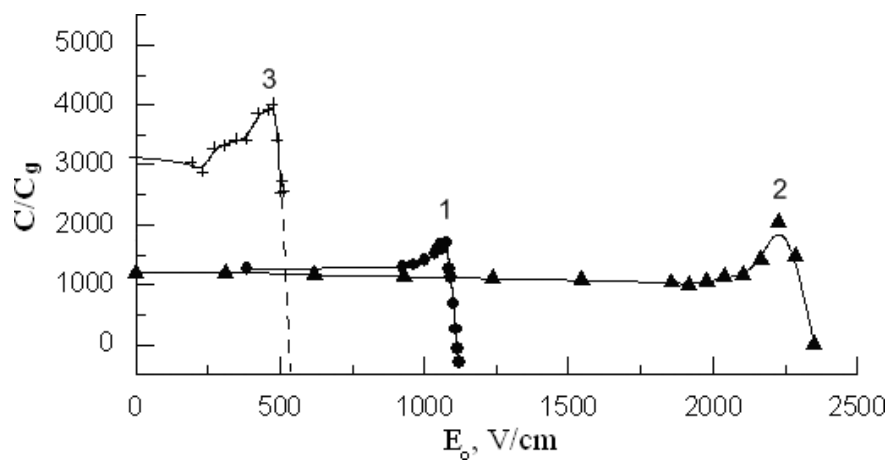

Fig. 3. Experimental dependences of dielectric permittivity for samples of the ceramics under consideration of types 1 (curve 1), 2 (curve 2) and 3 (curve 3). The dashed line shows extrapolation of dependence 3 to the negative values.

capacitance of the sample was measured, the maximum capacitance tended to decrease until its complete disappearance (Fig. 1).

Influence of frequency and temperature on field dependence of the capacitance of the experimental varistor ceramics illustrate $C-V$ characteristics typical for them, which are shown in Figs. 2 and 3. With the growth of frequency, at which the capacitance of a sample is measured, decrease of the maximum of capacitance in area 2 occurs (see Introduction) (Fig. 2) down to its complete disappearance.

The rise of temperature, to the contrary, leads to its increase [Fig. 2(a)]. $I-V$ characteristics for the same sample shown in Fig. 2(b) are measured in the same conditions. As it can be seen, the ohmic segment of the $I-V$ characteristics can be matched with area 1 of the $C-V$ characteristics, where the capacitance faintly changes with the growth of applied voltage, weak nonlinear segment — with area 2 (growth of capacitance) and the beginning of the varistor segment - with area 3 (decrease of capacitance).

Their specific features are substantial differences of absolute values of both permittivity and electric field strength, at which the mentioned areas show up. One of the possible causes of the data shown in Fig. 3 is manifestation of structural factors in formation of the capacitance properties of varistor ceramics.

\section{Discussion}

Interrelation between the $I-V$ and $C-V$ characteristics noted in the previous clause indicates the egenerality of electron processes responsible for the field effects under consideration. Thus, field dependences of dielectric permittivity (or capacitance), can be interpreted in the framework of models of nonlinear conductance of the $I-V$ characteristic, ${ }^{25}$ including existing notions about irregularity of their structure. ${ }^{15-17}$

The range of voltages, in which the negative capacitance is observed, corresponds to the range of voltages of the varistor segment of the $I-V$ characteristics, which is the most
Table 2. Comparison of the magnitude of the electric field of transition of the capacity to negative values and the average size of the grains of $\mathrm{ZnO}$ in the examined types of ceramics.

\begin{tabular}{cccc}
\hline Type of ceramics & $a, \mu \mathrm{m}$ & $E(C=0), \mathrm{V} / \mathrm{cm}$ & $E(C=0) \cdot a, \mathrm{~V}$ \\
\hline 1 & 10 & 1120 & 1.12 \\
2 & $3-5$ & 2400 & $0.7 \ldots 1.18$ \\
3 & 22 & 550 & 1.10 \\
\hline
\end{tabular}

sensitive to the irregularity of the structure, in particular, to formation and change of its clusters causing percolation direct current conductivity.

Data in Table 2 show the presence of a correlation between the magnitude of the electric field corresponding to transition of the capacitance of a varistor structure to negative values $E(C=0)$ and the average size of grains of $\mathrm{ZnO} a$ in the ceramics examined in the research. (For the type 3 ceramic samples we could not achieve negative values of $C$ because of strong currents in this range of fields, which cause fast destruction of the samples).

Usage for these purposes of voltages at fixed current of the varistor (as it is done, for instance, for determination of the parameters of the technical threshold voltage ${ }^{19}$ ) is not always acceptable. These values, for different varistors, may go beyond the limits of the varistor segment (for instance, may correspond to the end of weakly linear segment of $I-V$ characteristics), whereas comparison of voltages of transition of the capacitance of the varistor to negative values provides more accurate "hitting" into the range of high nonlinearity of the varistor. And this, must provide higher credibility of implementation of physical mechanisms of electric transfer determining its varistor properties.

With the growth of the size of grains, the magnitude of the electric field $E(C=0)$ decreases. One may notice that the products $E(C=0) \times a$ of ceramics different in compositions and manufacturing techniques get very close values. Thus, the causes of the observed effect are related to the common peculiarities of the structure of ceramic objects.

Based on the general notions about percolation, electric conductance of irregular structures, crystals of $\mathrm{ZnO}$ are to be taken as the nodes of the basis (carrying) network, and intercrystallite potential barriers - as the links between the nodes. ${ }^{17}$

With the use of the results obtained in the framework of the Shklovskii-De Gennes model of links, ${ }^{26,27}$ an irregular structure can be characterized by the constant of the critical sub-network (by the correlation radius of an infinite cluster) $\Lambda$. This sub-network is represented by one-dimensional chains of inter crystallite potential barriers located at a certain average distance equal to the average size of a crystallite $a$. The highest (the critical) potential barrier in the one-dimensional chain determines its resistance. This allows us to link the average electric field $\left(E_{c}\right)$ in a ceramic sample with the average local field $\left(U_{b} / a\right)$ on a separate inter crystallite 
barrier by the following statement:

$$
E_{c}=\left(U_{b} / a\right) \cdot(a / \Lambda)=U_{b} / \Lambda,
$$

where $U_{b}$ is the voltage applied to this barrier (we can neglect the thickness of the intercrystallite barrier in comparison with the size of the grains).

It should be noted that the value $\Lambda$ depends on the strength of the electric field. In strong electric fields, with the growth of $E_{c}$ the value $\Lambda$ decreases. ${ }^{28}$ This corresponds to the notion that, in distribution of voltage applied to the above-mentioned chain the voltage drop is stronger on barriers with higher resistance (with larger height). Owing to the nonlinear $I-V$ characteristic of the intercrystallite barrier, with the increase of the voltage applied to the chain, the resistance (height) of these high-resistance barrier reduces more than that of other barriers with lower resistance (lower height). As a result, electric field changes the distribution of magnitudes (or heights) of the intercrystallite potential barriers. Less-resistant (lower) barriers become included into the number of the "threshold" barriers, even though they were not such in the absence of the electric field. As a result, the average distance (the correlation radius of the infinite cluster) between the "key" inter crystallite potential barriers reduces.

Correlation between the voltage $E_{c}(C=0)$ and the average size of a crystallite, as well as quantitative closeness of the products $E_{c}(C=0) \times a$ can be viewed as the consequence of the ability of a ceramic varistor structure to come to a higher level of uniformity for invoking the main mechanism of high nonlinearity of the $I-V$ characteristic - the Zener tunneling from the valence zone of one crystallite into the zone of conductance of the other (adjacent one). Taking into account that the minimum voltage on a critical intercrystalline potential barrier required for implementation of this mechanism of field emission is estimated as $\sim 3.2-3.5 \mathrm{~V},{ }^{29}$ according to (1) it is possible to evaluate the size of an infinite cluster in the order of 3 to 5 grains of $\mathrm{ZnO}$. Thus, in the framework of the Shklovskii-De Gennes model used here, in a one-dimensional chain forming the critical sub-network, there should be 3 to 5 potential barriers.

\section{Conclusions}

Data of research in $C-V$ characteristics of ceramic structures for commercial varistors are given. A relationship between the intensity of electric field corresponding to transition of the $C-V$ characteristics to negative capacitances and the sizes of the grains of a varistor structure is established.

Results of comparison of $C-V$ characteristics of varistor ceramic samples with different chemical compositions and manufacturing techniques allowed us to assume that capacitance properties of ceramics are substantially dependent on their electrical structural irregularity, in particular, on formation and change of clusters causing percolation direct current conductivity.
It is shown that on the highly nonlinear segment of the $I-V$ characteristic of a varistor structure, the size of the infinite cluster is limited by 3 to 5 intercrystallite potential barriers for the studied commercial ceramics.

\section{References}

${ }^{1} \mathrm{R}$. Einziger, Grain junction properties of $\mathrm{ZnO}$ varistors, Appl. Surf. Sci. 3, 391 (1999).

${ }^{2} \mathrm{G}$. E. Pike, Electronic properties of $\mathrm{ZnO}$ varistors: A new model, Grain boundaries in semiconductors, Mat. Res. Soc. Symp. Proc. 5, 369 (1982).

${ }^{3}$ V. B. Kvaskov, Semiconductors with Bipolar Conductivity (Energoatomizdat, Moscow, 1988), p. 128 (in Russian).

${ }^{4}$ K. Mukae, K. Tsuda and I. Nagasawa, Capacitance-vs-voltage characteristics of $\mathrm{ZnO}$ varistors, J. Appl. Phys. 50(6), 4475 (1979).

${ }^{5}$ P. R. Bueno, M. R. de Cassia-Santos, E. R. Leite, E. Longo, J. Bisquert, G. Garcia-Belmonte and F. Fabregat-Santiago, Nature of the Schottky-type barrier of highly dense $\mathrm{SnO}_{2}$ systems displaying nonohmic behavior, J. Appl. Phys. 88(11), 6545 (2000).

${ }^{6}$ M. H. Wang, Q. H. Tang and C. Yao, Electrical properties and AC degradation characteristics of low voltage $\mathrm{ZnO}$ varistors doped with $\mathrm{Nd}_{2} \mathrm{O}_{3}$ Ceram. Int. 36(3), 1095 (2010).

${ }^{7}$ H. J. Long, W. J. Hu and J. Liu, Nickel oxide doping effects on electrical characteristics and microstructural phases of $\mathrm{ZnO}$ varistors with low residual voltage ratio, J. Ceram. Soci. Japan 119 (1385), 43 (2011).

${ }^{8} \mathrm{C}$. W. Nahm, Major effects on varistor properties of $\mathrm{ZnO}-\mathrm{V}_{2} \mathrm{O}_{5}-$ $\mathrm{MnO}_{2}-\mathrm{Nb}_{2} \mathrm{O}_{5}-\mathrm{Er}_{2} \mathrm{O}_{3}$ ceramics with sintering changes, Ceram. Int. 38(3), 2593 (2012).

${ }^{9}$ T. Y. Zheng, Z. X. Li, Q. Wang and M. Luo, Grain-Boundary and thermally stimulated current characteristics of $\mathrm{Y}_{2} \mathrm{O}_{3}$ doped $\mathrm{ZnO}$ varistor, J. Ame. Ceram. Soci. 96(11), 3518 (2013).

${ }^{10}$ G. D. Mahan, L. M. Levinson and H. R. Philipp, Theory of conduction in ZnO varistors, J. Appl. Phys. 50(4), 2799 (1979).

${ }^{11} \mathrm{~L}$. F. Lou, Semiconducting properties of $\mathrm{ZnO}$-grain-boundary$\mathrm{ZnO}$ junction in ceramic varistors, Appl. Phys. Lett. 36(7), 570 (1980).

${ }^{12}$ G. Blatter and F. Greuter, Electrical breakdown at semiconducting grain boundaries, Phys. Rev. B 34, 8555 (1986).

${ }^{13}$ L. M. Levinson, $\mathrm{ZnO}$ varistor technology, Mater. Eng.-New York 25, 431 (2004)

${ }^{14}$ A. B. Glot, A. V. Gaponov and A. P. Sandoval-García, Electrical conduction in $\mathrm{SnO}_{2}$ varistors, Physica B: Conden. Matter 405(2), 705 (2010).

${ }^{15}$ C. W. Nan, X. Zhou, J. Nan and J. Wu, Grain-boundary-controlled impedances of electroceramics: Generalized effective-medium approach and brick-layer model, J. Appl. Phys. 89, 3955 (2001).

${ }^{16}$ M. Bartkowiak, G. D. Mahan, F. A. Modine, M. A. Alim, R. Lauf and A. McMillan, Voronoi network model of $\mathrm{ZnO}$ varistors with different types of grain boundaries, J. Appl. Phys. 80(11), 6516 (1996).

${ }^{17}$ A. S. Tonkoshkur, Percolation effects in the electrical conductivity of a zinc oxide base varistor ceramic, Sov. Phys. - Solid State 32(8), 1313 (1990).

${ }^{18}$ A. S. Tonkoshkur, Volt-farad characteristic of inhomogeneous structure, Ukrainskii fizicheskii zhurnal 26(10), 1740 (1981) (in Russian). 
${ }^{19}$ I. V. Gomilko, A. S. Tonkoshkur and V. O. Makarov, Complex model of conductivity and dielectric constant field dependences of the zinc oxide ceramics, Br. Ceram. Proc. 60, 117 (1999).

${ }^{20} \mathrm{~A}$. I. Ivon, Parameters of $\mathrm{ZnO}$ grains in $\mathrm{ZnO}$-based ceramics, Inorg. Mater. 36(10), 1074 (2000).

${ }^{21}$ Z. Y. Lu, A. B. Glot, A. I. Ivon and Z. Y. Zhou, Electrical properties of new tin dioxide varistor ceramics at high currents, J. Eur. Ceram. Soc. 32(14), 3801 (2012).

${ }^{22} \mathrm{Z}$. Zivic, A multilayer chip varistors: The future in the low voltage transient suppression - part I: Fabrication and characterization, Informacije Midem 24, 161 (1994).

${ }^{23}$ V. A. Makarov, A. S. Tonkoshkur and I. V. Gomilko, Investigation of electronic processes at the grain boundaries of a multilayer varistor, Key Eng. Mater. 132, 1309 (1997).

${ }^{24}$ I. V. Gomilko, Polarization phenomena in oxide varistor ceramics. - Manuscript. Thesis for physical and mathematical science candidate"s degree on speciality 01.04 .07 - solid state physics. Dniepropetrovsk National University, Ukraine, Dniepropetrovsk (2001) (in Ukrainian).

${ }^{25}$ T. K. Gupta, Application of Zinc Oxide varistors, J. Am. Ceram. Soc. 73(7), 1817 (1990).

${ }^{26} \mathrm{P}$. G. de Gennes, On a relation between percolation theory and the elasticity of gels, Journal de Physique Lettres 37(1), 1 (1976) (in French).

${ }^{27}$ B. I. Shklovskii and A. L. Efros, Electronic Properties of Doped Semiconductors, (Springer, Heidelberg, 1984).

${ }^{28}$ B. I. Shklovskii, Percolation conductivity in strong electric fields, Sov. Phys. Semicond. 13, 53 (1979).

${ }^{29}$ E. Olsson and G. L. Dunlop, Characterization of individual interfacial barriers in a $\mathrm{ZnO}$ varistor material, J. Appl. Phys. 66(8), 3666 (1989). 